\title{
Prosthetic Rehabilitation of a Lateral Midfacial Defect Combined with Hemimandibulectomy using Multiple/ Sectional Prosthesis
}

\author{
${ }^{1} \mathrm{RM}$ Geethu, ${ }^{2} \mathrm{~S}$ Anilkumar, ${ }^{3} \mathrm{C}$ Rajesh, ${ }^{4}$ Shashank Uniyal
}

\begin{abstract}
Extraoral maxillofacial rehabilitation of patients with large midfacial defects has always perplexed the prosthodontist. Retention of such large facial prosthesis can be challenging due to its increased size and weight. This clinical report describes the technique of prosthetic rehabilitation of a patient with large midfacial defect using a hollow light-weight intraoral-extraoral combination prosthesis utilizing rare-earth magnets as a retention tool. This prosthesis dramatically improved the function, esthetics and comfort, thus, enabling him to lead a normal life.
\end{abstract}

Keywords: Midfacial defects, Intraoral-extraoral combination prosthesis, Rare earth magnets, Magnetically retained.

How to cite this article: Geethu RM, Anilkumar S, Rajesh C, Uniyal S. Prosthetic Rehabilitation of a Lateral Midfacial Defect Combined with Hemimandibulectomy using Multiple/Sectional Prosthesis. Int J Prosthodont Restor Dent 2014;4(4):131-137.

\section{Source of support: Nil}

Conflict of interest: None

\section{INTRODUCTION}

Man's need for restoring natural appearance of missing body parts undoubtedly dates back as far as humanity itself. ${ }^{1}$ Oral cancer is one of the 10 leading cancers in the world, causing destruction of structures, including mandible, maxilla, floor of the mouth and tongue, which often requires surgical management and prosthetic rehabilitation. $^{2-4}$

Midfacial defects are defined as defects in the middle third of the face in horizontal plane that communicate with intraoral maxillary defects. ${ }^{5}$ Marunick et al classified these defects into three categories, i.e. midline midfacial defects, lateral midfacial defects and combinations of these two. Midline midfacial defects are those defects which include complete or partial involvement of either

\footnotetext{
${ }^{1}$ Junior Resident, ${ }^{2}$ Professor and Head, ${ }^{3}$ Assistant Professor ${ }^{4}$ Senior Resident

${ }^{1-4}$ Department of Prosthodontics, Government Dental College, Kottayam, Kerala, India
}

Corresponding Author: RM Geethu, Junior Resident Department of Prosthodontics, Government Dental College Kottayam, Kerala, India, Phone: 9387085250, e-mail: drgeethumini@gmail.com nose or upper lip that communicate with an intraoral maxillary defect while lateral midfacial defects are those defects which include complete or partial involvement of cheek and orbital contents that communicate with an intraoral maxillary defect. ${ }^{6}$ Midfacial defects may result from either congenital or developmental abnormalities, accidental trauma or acquired disfigurements. Acquired midfacial defects often lead to severe facial disfigurement, impaired speech and swallowing difficulty, thus adversely affecting the patient's quality of life, psychology and social behavior. ${ }^{7-10}$

Despite advances in surgical procedure, surgical reconstruction alone cannot restore the esthetic and functional requirements of such patients as much as a facial prosthesis. ${ }^{5}$ The prosthetic rehabilitation is often more advantageous, since the diseased area is visible and can be inspected. Besides this, since the surgical and hospitalization time is shortened, the treatment cost is also reduced and thus allows the patient to be psychosocially reintegrated more quickly. ${ }^{11}$

Mutual retention of the intra- and extraoral prosthesis can be successfully achieved through magnets. The introduction of rare-earth permanent magnets made of alloys, such as neodymium-ferro-boron and samariumcobalt has resulted in magnets of very small dimensions. A common problem associated with use of magnets is tarnish and corrosion. It can be overcome by coating these magnets with nickel, gold and titanium. ${ }^{12}$

Restoration of large midfacial defects is a challenge to the artistic ability of the prosthodontist, moreover securing this prosthesis in place can be a formidable task because of its size and weight. ${ }^{13,14}$ This clinical report describes the technique of prosthetic rehabilitation of a patient with large midfacial defect using a hollow lightweight intraoral-extraoral combination prosthesis utilising rare-earth magnets as a retention tool.

\section{CASE REPORT}

A 59 years old male reported to the Department of Prosthodontics, Government Dental College, Kottayam, for prosthetic rehabilitation following right maxillectomy for squamous cell carcinoma of maxilla done 6 months 


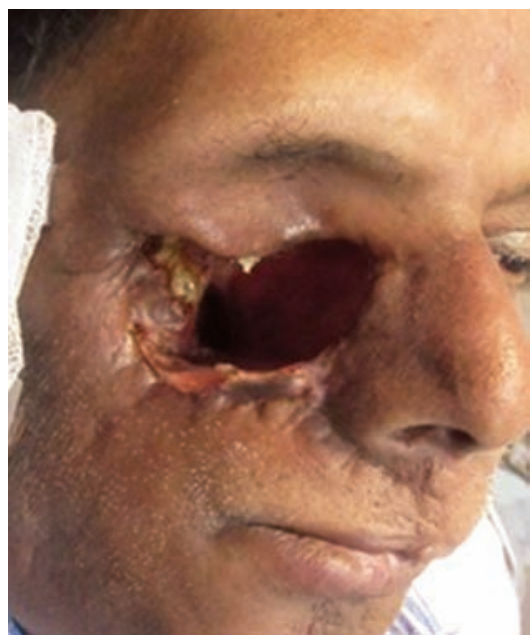

Fig. 1: Pretreatment extraoral view

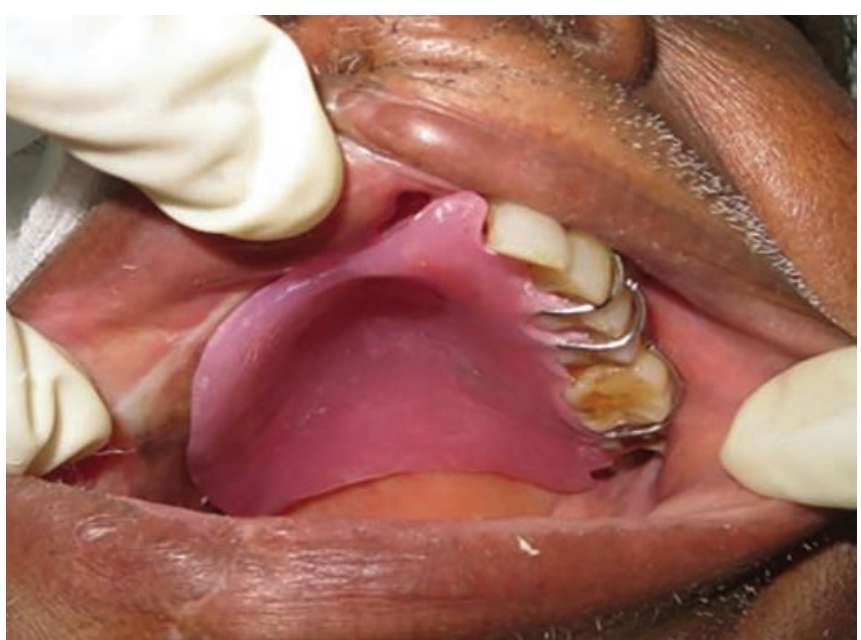

Fig. 3: Intraoral obturator prosthesis

back. Intraoral examination revealed a subtotal right maxillectomy defect and partially edentulous maxilla of the left side. The defect corresponded to the Aramany's class I situation. The defect was rehabilitated with a conventional interim closed hollow bulb obturator.

The patient reported back after 6 months to Government Medical College, Kottayam, with recurrence exhibiting a large tumor over the right maxillary region extending from the right lower eyelid (pushing the eye into the orbit) onto the lateral wall of right nose and posteriorly to the molar region, intraorally. He underwent radical maxillectomy including right maxillary sinus, right hemimandibulectomy, excision of right orbit from the base of skull and primary reconstruction with flap. Following postoperative radiation the buccal cover part of the flap got necrosed while leaving the palatal part intact, resulting in a huge defect. Patient was once again referred for prosthetic rehabilitation.

Extraoral examination revealed a postsurgical defect on the right side of the face extending from midline to the cheek which was in continuation with the palatal defect intraorally. Margins of the defect and tissues

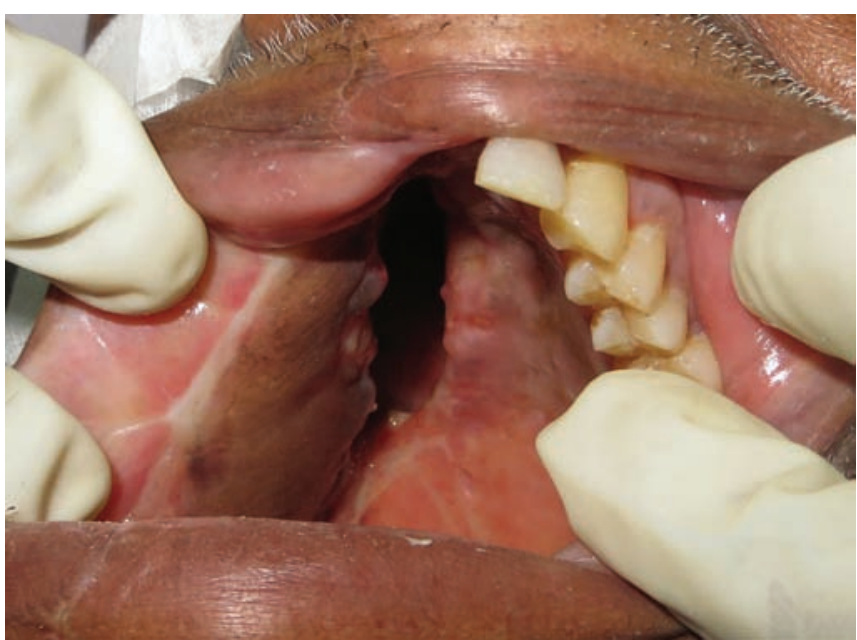

Fig. 2: Pretreatment intraoral view

within were normal and healthy. Though the patient was partially edentulous, periodontal condition of the remaining maxillary and mandibular teeth were satisfactory. Temperomandibular joint examination revealed deviation of mandible to the resected side with limited range of mandibular movements (Figs 1 and 2). Deglutition and speech were drastically affected and patient was restricted to liquid diet through the attached nasogastric tube.

Due to extensive size of the defect and minimal bony support, prosthetic rehabilitation was planned with a magnet-retained intraoral-extraoral combination prosthesis. Treatment procedure was planned to be completed in the following four stages:

- Stage I: Fabrication of obturator part

- Stage II: Fabrication of guiding flange

- Stage III: Fabrication of antral prosthesis

- Stage IV: Fabrication of facial prosthesis

\section{Fabrication of Obturator Part}

Preliminary impression of the maxillary arch along with the palatal defect was made using irreversible hydrocolloid impression material (Tropicalgin, Zhermack, Italy). Care was taken to record the maximum extent of the maxillary defect while limiting the flow of the material into the floor of the orbital defect. Impression was poured in type III dental stone (Gypstone, Prevest Denpro Ltd., Jammu, India). Defect was rehabilitated with conventional hollow bulb obturator, fabricated in heat polymerized polymethyl methacrylate (PMMA) resin (Pyrax Polymers, Roorkee, India) using the lost salt technique. The Prosthesis was retained with clasps on 21, 24, 26 (Fig. 3). After removing the nasogastric tube, obturator was inserted and compression areas were relieved. Patient was instructed to report after one week for review. On review, patient was found comfortable 


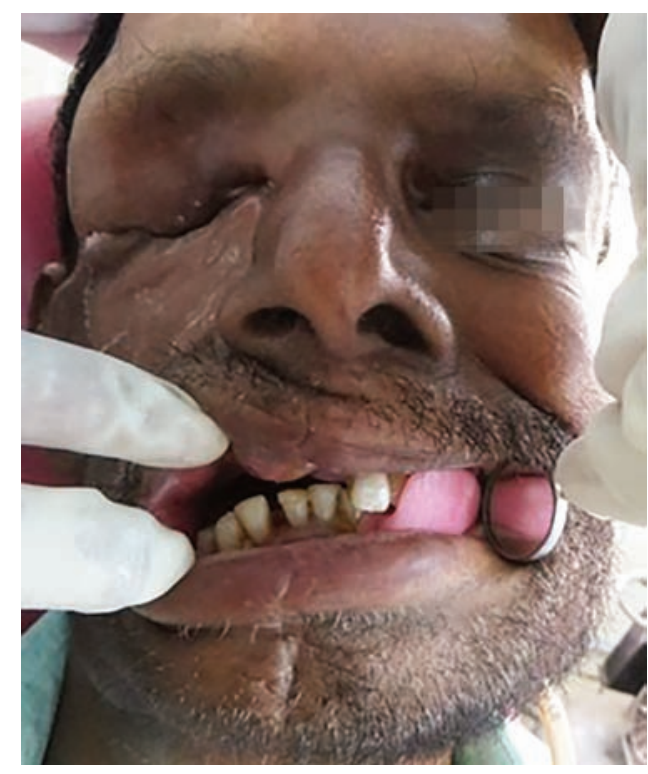

Fig. 4: Guiding flange prosthesis in place

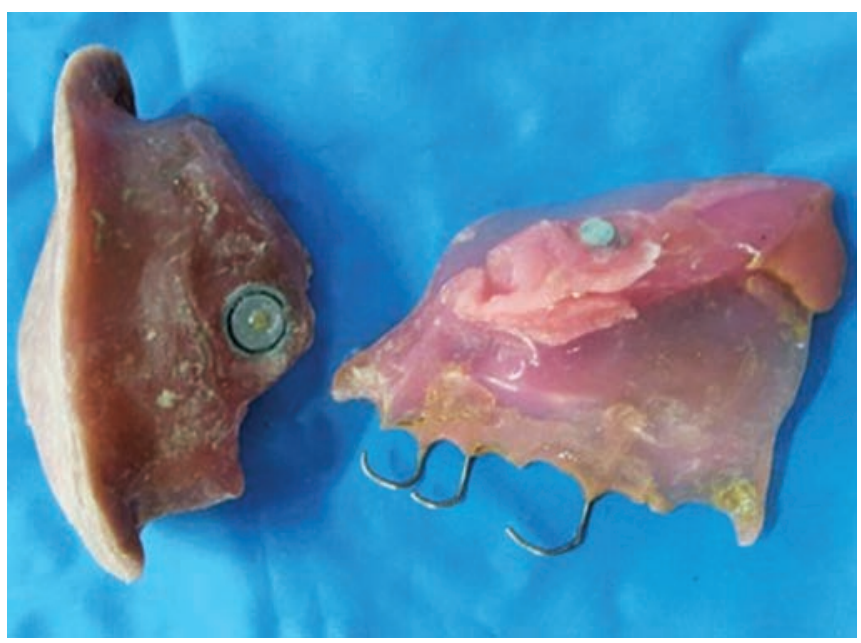

Fig. 6: Obturator and antral prosthesis embedded with magnets

with improved swallowing and speech so that the second stage of rehabilitation (i.e. guiding flange) was planned.

\section{Fabrication of Guiding Flange}

As the mandible could be manually guided into centric occlusion, guiding flange prosthesis was planned to reduce the mandibular deviation. Definitive impressions were made with irreversible hydrocolloid for maxilla and mandible, and casts were poured with type III dental stone. A 19-gauge stainless steel orthodontic wire was manipulated to fabricate a substructure and acrylised in autopolymerising PMMA resin (DPI-RR, Dental Products of India) (Fig. 4). Patient was advised to use the prosthesis throughout the day, except at night and during meals and was followed up at weekly intervals for the next 1 month.

\section{Fabrication of Antral Prosthesis}

After using the guiding flange for 1 month, the patient was able to close into maximum intercuspal position with

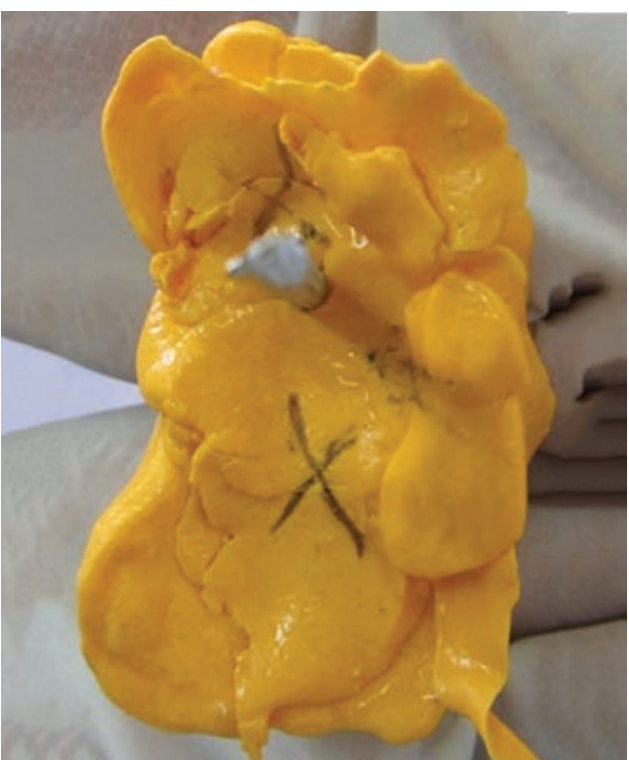

Fig. 5: Three-piece putty impression of the extraoral defect

the obturator in place. Impression of the extraoral defect was recorded with putty consistency polyvinyl siloxane impression material (Elite-HD, Zhermack, Italy). Moist gauze was packed to prevent flow of the impression material into the undesired areas. Impression was made in three pieces for ease of retrieval, and orientation lines were marked. Once the material was set, these three parts were removed and reoriented externally (Fig. 5). Beading and boxing was done, and cast poured in type III dental stone. A single thickness base plate wax (Hindustan Modeling Wax no. 2, Hyderabad) was adapted over the surface of the cast and was processed in heat polymerizing PMMA resin to obtain a hollow open prosthesis. A lid was fabricated in autopolymerising PMMA resin by lost salt technique, thus obtaining a hollow antral prosthesis. The hollow prosthesis was relined with silicone softliner (Molloplast-B, Detax GmbH \& Co, KG, Germany) in the undercut areas for ease of insertion and removal.

A pair of cobalt-samarium magnets $3 \mathrm{~mm}$ in diameter and $2.5 \mathrm{~mm}$ in thickness was selected for mutual retention between intraoral and extraoral prostheses (Fig. 6). One of the magnets was embedded in the superolateral aspect of the obturator plate by using autopolymerizing PMMA resin (Fig. 7). Counter position of the magnet in the antral prosthesis was traced by using pressure indicating paste. Magnets with opposite pole were incorporated in the inferomedial surface of the antral prosthesis (Fig. 8). Patient was reviewed regularly for 6 weeks and was found comfortable.

\section{Fabrication of Facial Prosthesis}

An irreversible hydrocolloid facial moulage was made with the antral prosthesis in situ. A wax box was fabricated to limit the flow of the impression material and a 


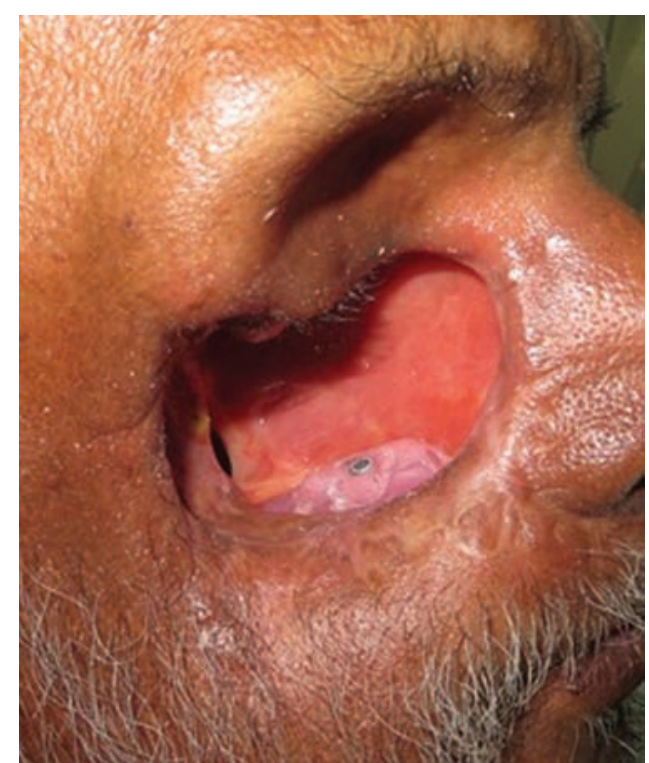

Fig. 7: Intraoral obturator prosthesis with magnet

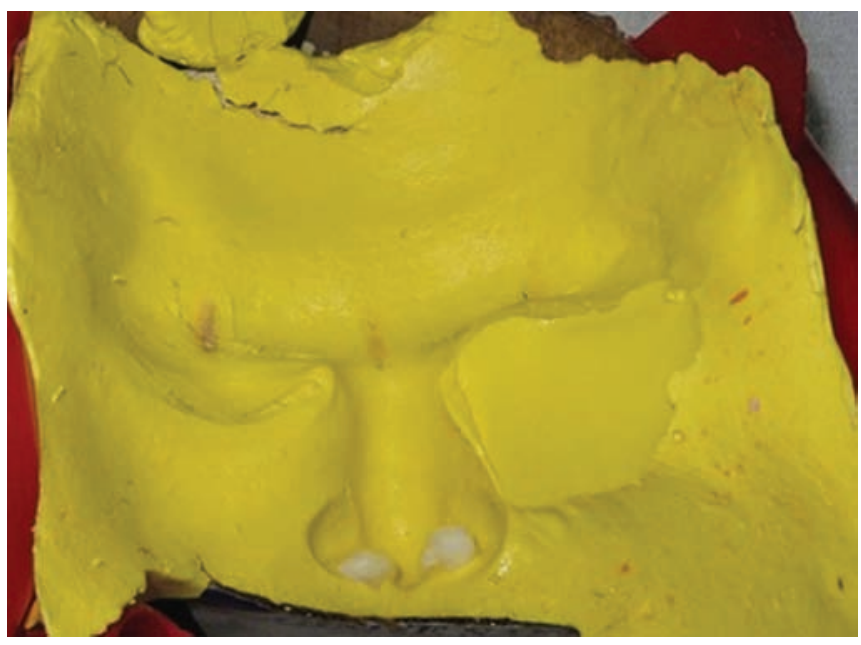

Fig. 9: Facial moulage

$1 "$ thick plastic tube was placed in the mouth to facilitate respiration during impression procedure (Fig. 9). A mold of type III dental stone was poured from the impression (Fig. 10).

A prefabricated stock eyeshell was selected to match size, shape and color of the normal left eye. To orient the eyeshell, patient was asked to gaze straight ahead; distance from the pupil of the normal eye to the midline was used in establishing the horizontal position and vertical position by the canthus relationship.

The facial portion of the prosthesis was sculpted in hard wax (Cavex Setup Regular, Cavex Holland BV, Netherlands). The fitting of the wax pattern was evaluated along with the orientation of the eyeshell and the facial contours as compared with the contralateral side (Figs 11 and 12). Two-piece mold was fabricated in type II gypsum (Kaldent, Kalabhai Karson Private Ltd., Mumbai) for packing silicone (Fig. 13). Shade matching was done in natural day light. Mold was packed with room temperature

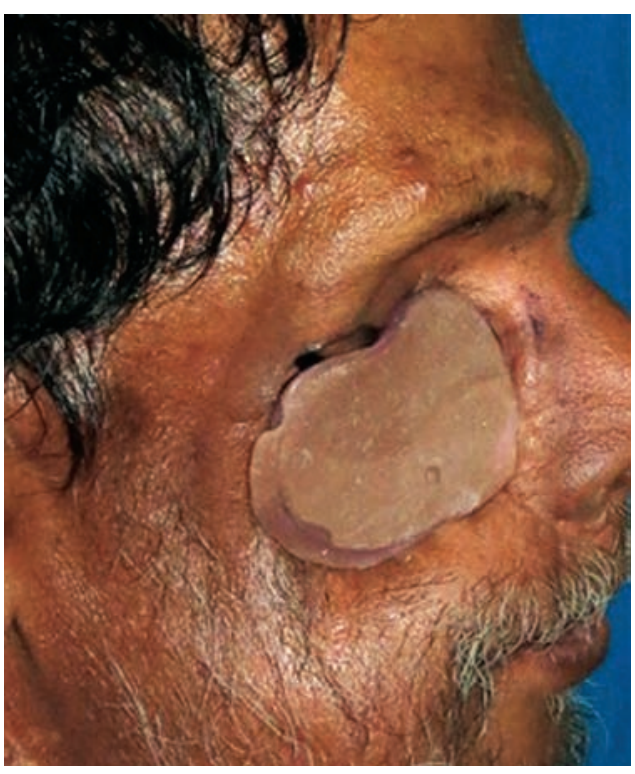

Fig. 8: Antral prosthesis

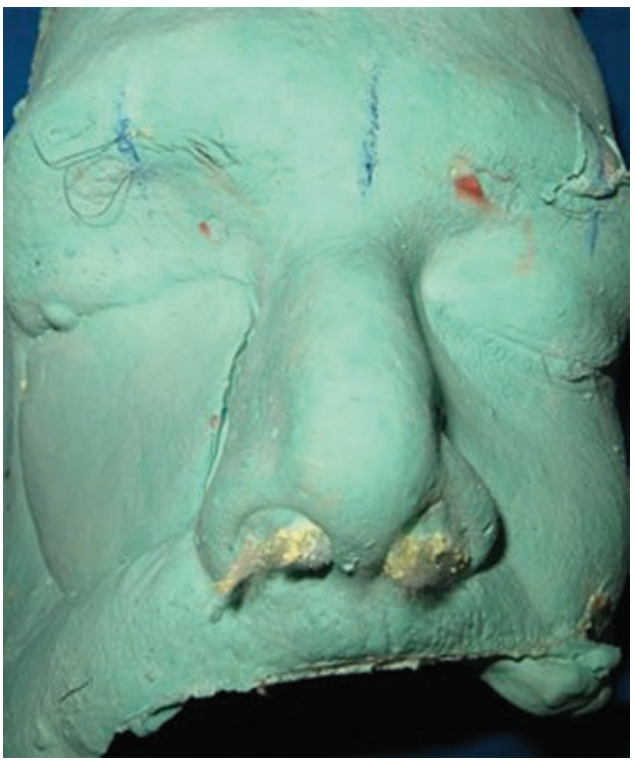

Fig. 10: Working cast

vulcanization silicone (Factor II, USA) with added colors to achieve appropriate characterization. Curing was done for 30 minutes at room temperature. Once the final prosthesis was retrieved, flash was trimmed and prosthesis was finished. Extrinsic pigmentation was done in the presence of patient. Eyelashes and mustaches were added by needle prick technique. The extraoral silicone prosthesis was attached to the hollow antral prosthesis with the help of cyanoacrylate adhesive (Fig. 14).

Patient was advised to insert the obturator portion first followed by the extraoral prosthesis and vice versa during removal. Instructions regarding maintenance of silicone prosthesis were also given. Separate eyeglasses were given to support and camouflage the prosthesis margins (Fig. 15). Patient was reviewed after 1 week. As the prosthesis was being placed on resilient soft tissue, marginal adaptation was compromised. Hence, 


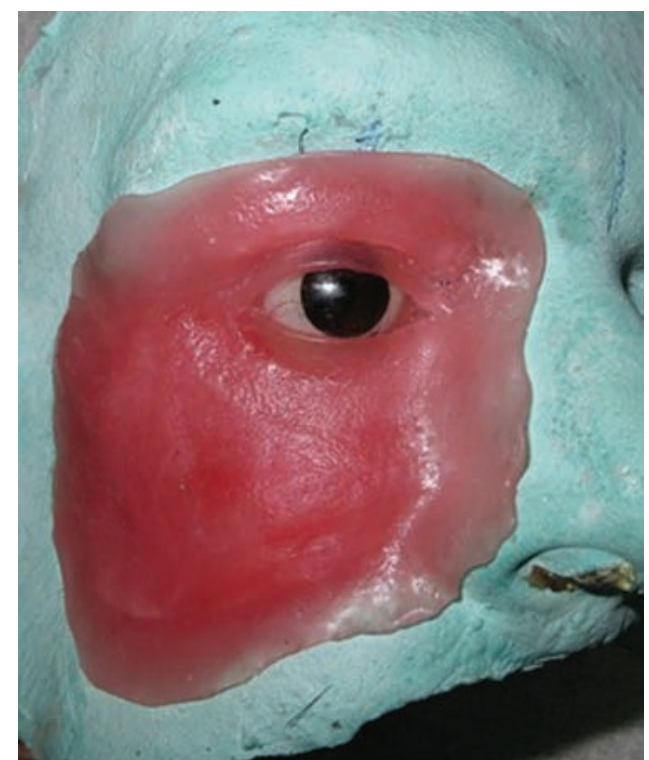

Fig. 11: Wax pattern

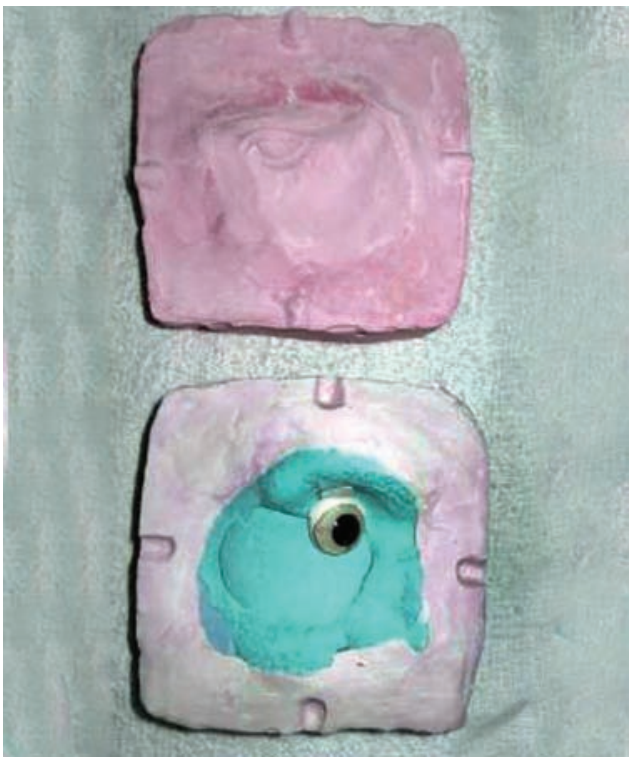

Fig. 13: Two-piece mold

patient was taught to use silicone medical adhesive and instructions were given regarding its frequency of use and maintenance. Regular follow-ups were done every 4 to 6 months.

\section{DISCUSSION}

Rehabilitation of patients with maxillofacial defects has always remained a challenge for the prosthodontist. Restoration of midfacial defects can be accomplished surgically, prosthetically or a combination of both methods; selection of which depends on many factors including size, location of the defect and age of the patient. ${ }^{15}$

Silicones have been used for over 50 years in the field of maxillofacial prosthetics, with desirable material properties, including flexibility, biocompatibility, ability to accept intrinsic and extrinsic colorants, chemical and

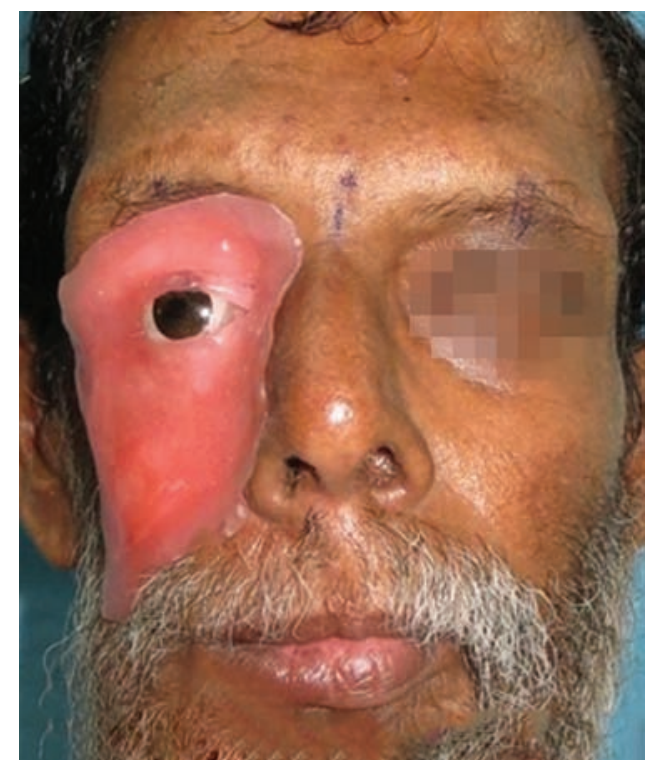

Fig. 12: Wax pattern try-in

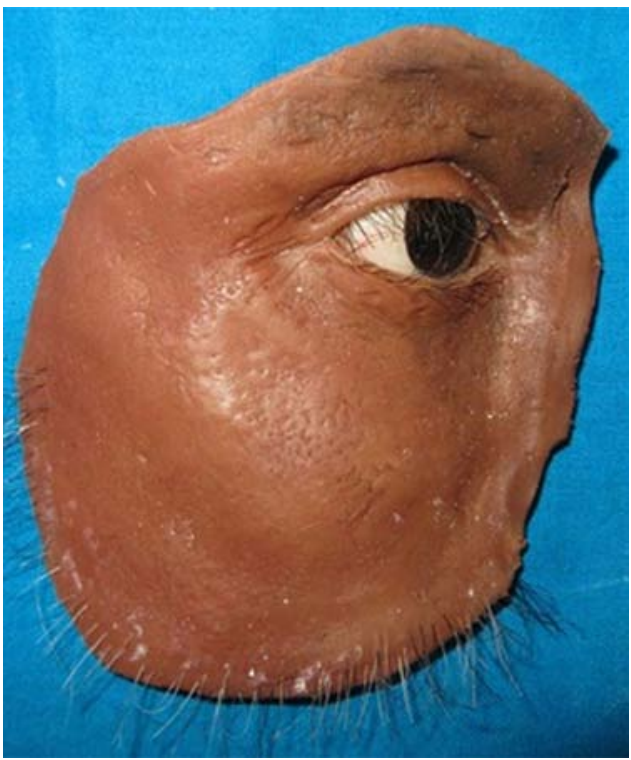

Fig. 14: Finished prosthesis

physical inertness and moldability. ${ }^{14}$ Room temperature vulcanising silicones had been used in this case to achieve a life-like appearance. Heat-cured polymethyl methacrylate was used for fabrication of the intraoral prosthesis because of its biocompatibility and ability to bond with soft permanent liner. But, candidal adherence on the soft liner, guarded color stability and durability of the silicone can affect the longevity of the prosthesis.

Increased weight of the PMMA framework is a worry for the prosthodontists. Fiber-reinforced composite was introduced as a dental and medical biomaterial for the fabrication of light-weight facial prosthesis framework. But, cost and technique sensitivity of this material has limited its use in dentistry. ${ }^{16}$ This article describes a technique to make a light-weight PMMA substructure by making it hollow. Many methods and materials have 


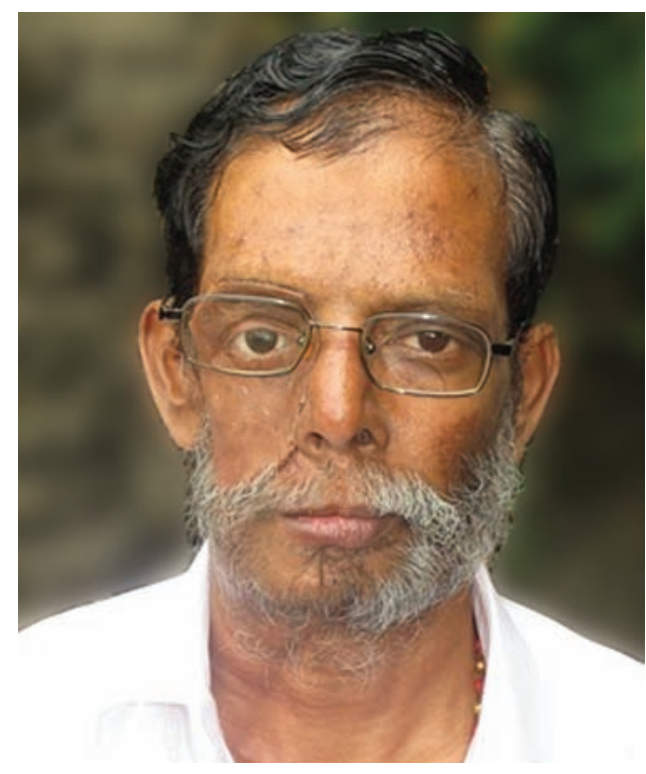

Fig. 15: Post-treatment extraoral view

been used to fabricate a hollow PMMA framework, such as sugar ${ }^{17}$ and ice. ${ }^{18}$ In this case, light-weight prosthesis was fabricated using the lost salt technique, which not only helped to combat retention problems due to gravity but also enhanced the resonance of speech.

The cast-metal framework improves retention, stability, support and bracing of the prosthesis and, thus, increases the longevity of both prosthesis as well as supporting tissues. In this case, cast-metal framework was avoided due to possibility for recurrence of lesion, complex clinical and laboratory procedures ${ }^{16}$ and cost factors.

Even though acceptable cosmetic results usually can be obtained, retention of a large prosthesis can be challenging. Various methods of auxiliary retention for facial prosthesis have been described in the literature; they include eyeglasses, denture extensions that engage tissue undercuts, magnets, facial prosthetic adhesives or combination of the above and craniofacial implants. ${ }^{19-22}$ Although osseointegrated implants provide the most reliable prosthesis retention: additional surgeries, expenses, inadequate bone and prior radiation to the area contraindicated this type of treatment. For the first time, Nadeau ${ }^{23}$ described the use of combination of extraand intraoral prostheses connected by magnets. These magnets are biocompatible and the inert magnetic field is harmless to human body and can be safely used for patients with cardiac pacemaker. There may be a gradual decrease in retentive force upon repeated usage. Some open field uncoated magnet exhibits significant cytotoxic effects due to the release of corrosion by products from them. Therefore, magnets should be replaced as early as signs of corrosion develop. ${ }^{24} \mathrm{~A}$ two-component prosthesis retained with the help of rare earth magnets was used in this case. Medical-grade silicone adhesive was used to obtain better marginal adaptation of silicone prosthesis with the adjacent skin of the patient. ${ }^{11}$

The advantage of sectional prosthesis was that all the three parts can be removed. This eliminated the need to fabricate the whole prosthesis again in case of discoloration or damage of the silicone layer..$^{25}$ The advantage of this prosthesis is that it is noninvasive, cost-effective, tissue tolerant, esthetic and comfortable to the patient. One of the major drawbacks of sectional prosthesis is that it does not allow for any margin of error of dead space between the magnets which can reduce the retentive force.

\section{CONCLUSION}

Satisfactory functional and esthetic results are achievable in patients with large lateral midfacial defect using hollow acrylic resin framework and silicone facial prosthesis. Retention of facial prosthesis can be enhanced with the use of strong magnets. In spite of several inherent limitations and complexity of this treatment procedure, a dedicated effort on the part of prosthodontist can always help to rehabilitate these unfortunate patients to the utmost satisfaction.

\section{REFERENCES}

1. Parr GR, Gardner LK. The evolution of the obturator framework design. J Prosthet Dent 2003;89(6):608-610.

2. Petersen PE. Strengthening the prevention of oral cancer: the WHO perspective. Community Dent Oral Epidemiol 2005;33(6):397-399.

3. Pisani P, Bray F, Parkin DM. Estimates of the world-wide prevalence of cancer for 25 sites in the adult population. Int J Cancer 2002;97(1):72-81.

4. Jemal A, Thomas A, Murray T, Thun M. Cancer statistics, 2002. CA Cancer J Clin 2002;52(1):23-47.

5. Brignoni R, Dominici JT. An intraoral-extraoral combination pros 2. Thesis using an intermediate framework and magnets: a clinical report. J Prosthet Dent 2001;85(1):7-11.

6. Marunick MT, Harrison R, Beumer J. Prosthodontic rehabilitation of midfacial defects. J Prosthetic Dent 1985;54(4): 553-560.

7. Ludlow JB, Davies-Ludlow LE, Brooks SL, Howerton WB. Dosimetry of 3 CBCT devices for oral and maxillofacial radiology: CB Mercuray, New Tom 3G and i-CAT. Dentomaxillofac Radiol 2006;35(4):219-226.

8. The glossary of prosthodontic terms. J Prosthetic Dent 2005; 94(1):10-92.

9. Lascala CA, Panella J, Marques MM. Analysis of the accuracy of linear measurements obtained by cone beam computed tomography (CBCT-NewTom). Dentomaxillofac Radiol 2004; 33(5):291-294.

10. Hecker DM, Wiens JP, Cowper TR, et al. Can we assess quality of life in patients with head and neck cancer? A preliminary report from the American Academy of Maxillofacial Prosthetics. J Prosthetic Dent 2002;88(3):344-351.

11. Pattanaik S, Wadkar AP. Rehabilitation of a patient with an intraoral prosthesis and an extraoral orbital prosthesis 
retained with magnets. J Ind Prosthodont Soc 2012 Jan-Mar; 12(1):45-50.

12. Drago CJ. Tarnish and corrosion with the use of intraoral magnets. J Prosthet Dent 1991;66(4):536-540.

13. Goiato MC, Fernandes AU, dos Santos DM, Positiol BVA. Ning magnets on a multiple/sectional maxillofacial prosthesis. J Contemp Dent Pract 2007;8(7):101-107.

14. Nair A, Regish KM, Shah FK, Prithviraj DR. Reconstruction of a midfacial defect using an intraoral-extraoral combination prosthesis employing magnets: a clinical report. J Clin Exp Dent 2012;4(3):e186-188.

15. Taylor TD. Clinical Maxillofacial Prosthetics, Quintessence, Carol Stream, Ill, USA; 2000.

16. Patil PG. Modified technique to fabricate a hollow lightweight facial prosthesis for lateral midfacial defect: a clinical report. J Adv Prosthodont 2010;2(3):65-70.

17. Matalon $\mathrm{V}$, LaFuente $\mathrm{H}$. A simplified method for making a hollow obturator. J Prosthet Dent 1976;36(5):580-582.

18. Schneider A. Method of fabricating a hollow bulb obturator, J Prosthet Dent 1978;40(3):351.
19. Thomas K. Prosthetic rehabilitation. London; Quintessence Publishing; 1994. p. 93-103.

20. Beumer J III, Curtis TA, Marunick MT. Maxillofacial rehabilitation: prosthodontic and surgical considerations. St Louis; Ishiyaku EuroAmerica Inc; 1996. p. 408-416.

21. Dumbrigue HB, Fyler A. Minimizing prosthesis movement in a midfacial defect: a clinical report. J Prosthet Dent 1997; 78(4):341-345.

22. Verdonck HW, Peters R, Vish LL. Retention and stability problems in a patient with a large combined intra- and extraoral defect: a case report. J Facial Somato Prosthet 1998; 4:123-127.

23. Nadeau J. Maxillofacial prosthesis with magnetic stabilizers. J Prosthetic Dentistry 1956;6(1):114-119.

24. Banerjee S, Kumar S, Bera A, Gupta T, Banerjee A. Magnet retained intraoral-extraoral combination prosthesis: a case report. J Adv Prosthodont 2012;4(4):235-238.

25. Hatami M, Badrian H, Samanipoor S, Goiato MC. Magnetretained facial prosthesis combined with maxillary obturator. Case Rep Dent 2013;406410. 